\title{
LUIZ MARCELLINO DE OLIVEIRA: APRENDENDO E ENSINANDO ANÁLISE DO COMPORTAMENTO
}

\author{
LUIZ MARCELLINO DE OLIVEIRA: LEARNING AND TEACHING BEHAVIOR ANALYSIS
}

\author{
SEBASTIÃO DE SOUSA ALMEIDA' \\ Faculdade de Filosofia, CIÊNCIAS E Letras de Ribeirão Preto, Universidade de SÃo PaUlo, Brasil
}

\begin{abstract}
RESUMO
Luiz Marcellino de Oliveira (1939-2008) foi um dos maiores entusiastas do ensino da análise experimental do comportamento no Brasil. Dedicou toda a sua vida profissional à tarefa de estudar, ensinar e difundir os conceitos da análise do comportamento. Sua incansável disposição para o ensino levou-o a participar de diversos fóruns relacionados à melhoria do ensino de psicologia no Brasil. Sua história se confunde a de criação e consolidação da Sociedade de Psicologia de Ribeirão Preto, antecessora da Sociedade Brasileira de Psicologia. Luiz de Oliveira foi pioneiro no estudo dos efeitos comportamentais da desnutrição no Brasil e fundador do Laboratório de Nutrição e Comportamento da Universidade de São Paulo, com grandes contribuições científicas para a área. O Centro de Estudo Multiprofissional de Apoio à Criança que procura recuperar atrasos no desenvolvimento produzidos por desnutrição, entre outros fatores de risco, existe hoje devido a sua iniciativa pioneira.

Palavras-chave: Luiz Marcellino de Oliveira, análise do comportamento, ensino de psicologia, desnutrição.
\end{abstract}

ABSTRACT
Luiz Marcellino de Oliveira (1939-2008) was one of the greatest enthusiasts of teaching behavioral analysis in Brazil. He dedicated his professional life to the task of learning, teaching and spread behavior analysis. His indefatigable disposition for teaching led him to participate in various forums dedicated to discuss the improvement of psychology teaching in Brazil. He was involved with the creation and consolidation of the Society of Psychology of Ribeirão Preto, predecessor to the Brazilian Psychology Society. Luiz de Oliveira was the pioneer in the area of behavior effects of malnutrition in Brazil and the founder of the Laboratory of Nutrition and Behavior at the University of São Paulo, making relevant scientific contributions to this area. The Multiprofessional Center for the Study and Support of the Children, dedicated to recover developmental impairments produced by malnutrition, among other risk factors, exists today as a result of his initiative.

Keywords: Luiz Marcellino de Oliveira, behavior analysis, psychology teaching, malnutrition.

1 Endereço para correspondência: FFCLRP-USP, Avenida Bandeirantes, 3900, Cep. 14040-901 Ribeirão Preto,SP, e-mail: sebasalm@usp.br. 
O Professor Luiz Marcellino de Oliveira (19392008), bacharel em psicologia pela Faculdade de Filosofia, Ciências e Letras de Ribeirão-USP, mestre e doutor em psicologia pela USP, com pós-doutorado pela Universidade de Cornell e Universidade do Chile, foi um dos maiores pioneiros na pesquisa e ensino da Análise Experimental do Comportamento em nosso país. Definitivamente não se pode falar da introdução e desenvolvimento da análise do comportamento no Brasil sem mencionar o nome de Luiz de Oliveira, como era conhecido pelos pesquisadores da área. Luiz de Oliveira começou seus estudos como aluno de graduação da Faculdade de Filosofia, Ciências e Letras de Rio Claro e, posteriormente, na Universidade de Brasília (UnB). Ainda em Brasília iniciou seus estudos de psicologia na UnB onde teve a oportunidade de acompanhar os esforços de Gilmour Sherman, Fred S. Keller, Carolina Martuscelli Bori e Rodolpho Azzi para implantar o curso de Psicologia, assim como o desenvolvimento e aplicação do Sistema Personalizado de Ensino proposto por Keller (Keller, 1996). Após um início promissor o grupo liderado por Carolina Bori e Fred Keller se dispersou em razão da crise enfrentada pela UnB durante a ditadura militar em 1965. Nesse período, Luiz de Oliveira decide deixar Brasília e se transferir para Ribeirão Preto, mais especificamente para a Faculdade de Filosofia, Ciências e Letras, que nessa época ainda era um Instituto Isolado de Ensino do Estado de São Paulo. Nessa faculdade concluiu seu curso de bacharelado em Psicologia e foi contratado como docente.

Após sua contratação pela Faculdade de Filosofia, Ciências e Letras de Ribeirão Preto em 1966, Luiz colocou em ação o projeto de instalação e manutenção de um núcleo de pesquisadores de Análise do Comportamento. Sob influência dos ensinamentos de Fred Keller iniciou o ensino de Análise do Comportamento aos alunos de graduação do curso de Psicologia utilizando o Sistema Personalizado de Ensino. O seu entusiasmo para aprender e ensinar os conceitos de análise do comportamento era tão grande que realiza todos os esforços para montar um laboratório de ensino de AEC no Departamento de Psicologia, laboratório esse que hoje se denomina Laboratório Fred S. Keller, uma iniciativa do próprio Luiz de Oliveira como forma de homenagear o grande mestre. Nesse laboratório Luiz de Oliveira ensinou Análise do Comportamento para alunos de graduação e pós-graduação em psicologia por mais de 40 anos. Já debilitado por sua doença ainda fez questão de participar do oferecimento da disciplina de graduação aos alunos do curso de psicologia no primeiro semestre de 2008.

Entre 1968 e 1971 desenvolveu seu Mestrado no Instituto de Psicologia da Universidade de São Paulo, em São Paulo, sob orientação da saudosa Profa. Dra. Carolina Bori, ainda no sistema antigo sem defesa de dissertação. Entre 1971 e 1973 desenvolveu seu Doutorado na Faculdade de Medicina de Ribeirão Preto sob orientação do Prof. Dr. Frederico Guilherme Graeff, sendo aprovado com a tese intitulada "Efeitos da anfetamina e apomorfina sobre o desempenho em intervalo fixo" (de Oliveira \& Graeff, 1972, Graeff \& de Oliveira, 1975). Já nesse período inicia uma interação com o Prof. Dr. José Eduardo Dutra de Oliveira da Faculdade de Medicina de Ribeirão Preto-USP, parceria essa que resultou nos primeiros estudos de Análise do Comportamento em animais desnutridos conduzidos no Brasil. Três anos após a conclusão de seu Doutorado Luiz de Oliveira viajou para os Estados Unidos onde deu seqüência aos estudos dos efeitos da desnutrição protéica precoce em modelos animais de Análise do Comportamento. Durante esses dois anos de pósdoutorado (1976-1977) Luiz de Oliveira desenvolveu pesquisa com um dos grupos mais atuantes no estudo dos efeitos comportamentais da desnutrição, grupo esse chefiado pelo Professor David A. Levitsky da Universidade de Cornell em Ithaca-USA (de Oliveira \& Levitsky, 1979).

Tive a oportunidade de, como aluno de graduação de psicologia ainda no primeiro ano, conhecer o Luiz de Oliveira em 1977 quando ele acabara de retornar de seu pós-doutorado nos Estados Unidos. Fui seu aluno no curso de Psicologia Geral e Experimental e conheci o seu entusiasmo como o ensinamento de Análise do Comportamento através do Sistema Personalizado de Ensino. Imediatamente também me entusiasmei com a área e com as novidades que o Luiz de Oliveira trazia do laboratório do Dr. David Levitsky, principalmente as idéias a respeito do estudo dos efeitos comportamentais da desnutrição protéica precoce. Conclui sua disciplina de graduação e fui convidado a fazer parte de seu grupo de pesquisa como aluno de iniciação científica em 1977. Com uma bolsa de iniciação científica da Fundação de Amparo à Pesquisa do Estado de São Paulo (FAPESP) fui orientando do Luiz de Oliveira até o final de meu curso de graduação. Tive, então, o privilégio de ser o primeiro aluno do grupo de pesquisa do Luiz de Oliveira a concluir um estudo sobre os efeitos da desnutrição protéica precoce na aprendizagem de uma resposta de esquiva em ratos. Esse primeiro trabalho desenvolvido no Laboratório de Nutrição e Comportamento também deu origem aos dois primeiros trabalhos do grupo publicados em revistas no exterior (Almeida \& De Oliveira, 1981, De Oliveira \& Almeida, 1985).

Nesse período fui testemunha, como monitor da disciplina de Psicologia Geral e Experimental, da paixão do Luiz de Oliveira com a formação do aluno de graduação. Essa paixão do Luiz pela formação do aluno acabou resultando em uma das experiências mais interessantes que conheço na formação científica em psicologia, que é o Programa de Bacharelado Especial do curso de Psicologia da USP Ribeirão Preto. Idealizado e concebido por ele, e com o apoio de vários colegas do Departamento de Psicologia, implantou-se um Programa de Bacharelado optativo onde os alunos de graduação, desde o segundo ano, têm a oportunidade de se engajar em um grupo de pesquisa chefiado por um dos docentes do Departamento. Durante esse Programa o aluno tem a oportunidade de planejar, executar e 
apresentar um trabalho de monografia ao final do curso de graduação. Apesar de optativo um número significativo de alunos de graduação em psicologia se engaja no Programa e concluem o Bacharelado com a apresentação da monografia. São inúmeros os relatos da importância desse Programa para a formação de nossos alunos, principalmente no que diz respeito à sua contribuição para os Programas de Pós-Graduação. Os alunos que participaram do Programa de Bacharelado Especial demonstram um desempenho diferenciado tanto na seleção quanto no desenvolvimento de seus programas de pós-graduação, mostrando a importância da iniciativa do Luiz de Oliveira na concepção do Programa.

Esse período, logo após o retorno dos Estados Unidos, coincidiu também com o projeto do Luiz de Oliveira de criação e implantação de um laboratório para o estudo dos efeitos comportamentais da desnutrição precoce em animais. Sua persistência e entusiasmo com o projeto, mesmo nas condições mais desfavoráveis de verba e espaço, contagiavam os alunos de seu grupo de pesquisa e deles teve o maior apoio na tarefa de construção desse laboratório. Desse esforço conjunto nasceu o Laboratório de Nutrição e Comportamento na USP-Ribeirão Preto, onde Luiz de Oliveira trabalhou até poucos dias antes de seu falecimento. A criação e implantação desse laboratório não foi tarefa fácil de ser executada. A Faculdade de Filosofia, Ciências e Letras de Ribeirão Preto acabara de ser incorporada ao Campus da Universidade de São Paulo e não dispúnhamos de infraestrutura física e material. Nesse período o Luiz de Oliveira reuniu um grupo de alunos do curso de graduação em Psicologia e começou a orientar projetos na área de nutrição e análise experimental do comportamento. Esse pequeno grupo de pioneiros do estudo da análise experimental do comportamento tinha que construir pessoalmente a maioria dos equipamentos a serem utilizados nos projetos de pesquisa orientados pelo Luiz de Oliveira. O próprio Luiz de Oliveira muitas vezes passava dias inteiros junto com os seus alunos de iniciação científica comprando, medindo, serrando e pregando madeiras para a construção de caixas experimentais. Terminadas as caixas experimentais era a vez do Luiz de Oliveira passar horas e horas montando racks para a colocação e programação de relés eletromecânicos utilizados no controle dos experimentos. Até os dias de hoje podemos encontrar, em uma pequena sala do laboratório, uma série desses aparelhos com os quais ele iniciou a implantação do Laboratório de Nutrição e Comportamento.

Seu entusiasmo pelo ensino e pela pesquisa logo deu frutos. Seu grupo de pesquisa cresceu rapidamente e, com o apoio institucional e as verbas de órgãos de fomento à pesquisa, o Luiz de Oliveira conseguiu montar um laboratório moderno e equipado. Nesse laboratório ele orientou 20 Dissertações de Mestrado, 10 Teses de Doutorado e 16 trabalhos de iniciação científica, além da orientação de diversas monografias de Bacharelado. Parte significativa desses orientandos está hoje contratada por instituições públicas e privadas de ensino superior, onde montou seus laboratórios e se dedicou a repassar ensinamentos adquiridos durante o período de orientação do Luiz de Oliveira.

Nesse laboratório também surgiram suas grandes contribuições para a área. Destacam- se os trabalhos publicados com modelos de exploração, modelos de aprendizagem (especialmente aqueles de discriminação complexa), modelos de memória, modelos de impulsividade e modelos de ansiedade. Deve-se também mencionar o interesse do Luiz de Oliveira pelo desenvolvimento de novas tecnologias para o estudo do comportamento, destacando-se aqui a construção de caixas experimentais para o estudo em abelhas, caixas experimentais para o estudo do padrão alimentar em ratos, caixas experimentais para o estudo de discriminações complexas, além de outras tantas para os estudos de exploração e ansiedade.

Em 1988, quando seu grupo de pesquisa já estava consolidado e ele já era reconhecido nacional e internacionalmente por seus estudos de análise do comportamento em animais desnutridos, o Luiz de Oliveira se lança a outro desafio. O desafio agora era estudar o comportamento de crianças desnutridas. Para tanto viaja em 1988 para desenvolver um segundo estágio de pós-doutorado na Universidade do Chile. Nessa universidade inicia seus estudos com humanos investigando os efeitos da desnutrição precoce no padrão de sono-vigilia de crianças. Essa experiência no Chile foi fundamental para o desenvolvimento de uma nova área de pesquisa em seu Laboratório de Nutrição e Comportamento, a área dos efeitos da estimulação ambiental na recuperação de prejuízos comportamentais e cerebrais causados pela desnutrição precoce. Os estudos nessa área culminaram na criação, através de uma Organização Não Governamental (ONG) do CEMAC Centro de Estudo Multiprofissional de Apoio à Criança. Nesse centro são atendidas crianças com atrasos no desenvolvimento, com o objetivo de avaliar os efeitos da estimulação ambiental (táctil e sinestésica) como estratégia para recuperar os atrasos produzidos por desnutrição, nascimento prematuro ou hipóxia ao nascer, entre outros fatores de risco.

Sua incansável motivação para a ciência ainda o conduziu para um último projeto para o Laboratório de Nutrição e Comportamento. Poucos meses antes de seu falecimento o Luiz de Oliveira iniciou um intercâmbio com o grupo do Prof. Dr. Leon Cintra do Instituto de Neurobiologia da Universidade Nacional Autônoma do México. Esse intercâmbio previa a colaboração da instituição mexicana na montagem de equipamentos para estudos neurofisiológicos de animais desnutridos no Laboratório de Nutrição e Comportamento da USPRibeirão Preto. Previa também o intercâmbio de alunos de pós-graduação para o desenvolvimento de pesquisas nos dois laboratórios. Infelizmente o Professor Luiz não teve tempo para concluir esse último projeto.

Porém, as contribuições do Luiz de Oliveira para a Psicologia não se restringem ao ensino e pesquisa. Extremamente relevante também foi sua atuação na difusão do conhecimento científico. Ele foi sócio-fundador da Sociedade de Psicologia de Ribeirão Preto e 
componente de sua primeira diretoria (1971-1973), atuando como segundo tesoureiro. A partir daí sua participação na consolidação dessa Sociedade e de sua sucessora a Sociedade Brasileira de Psicologia é conhecida por todos os pesquisadores da área de psicologia no Brasil. Foi presidente dessas duas sociedades por vários mandatos sempre com o mesmo entusiasmo dos primeiros anos. Quando não era presidente era comum encontrar o seu nome em outro cargo da diretoria sempre disposto a colaborar para o sucesso das diversas Reuniões Anuais da Sociedade. Esse espírito colaborativo na importante tarefa de divulgação científica foi largamente reconhecido pelos sócios da Sociedade Brasileira de Psicologia quando os associados aprovaram, por aclamação, a recomendação da concessão do título de Associado Honorário a Luiz de Oliveira, em solenidade em Ribeirão Preto no dia 17 de dezembro 2007. Nessa solenidade o Prof. Dr. José Lino de Oliveira Bueno, colega de longa data do Luiz de Oliveira no Departamento de Psicologia da USP-Ribeirão Preto foi muito feliz em afirmar que: (...) é difícil se falar da Análise do Comportamento no Brasil, da Sociedade Brasileira de Psicologia e dos estudos de Nutrição e Comportamento sem referência central a Luiz de Oliveira. Mais ainda, é impossível falarmos de Luiz de Oliveira sem que sejamos envolvidos por sua paixão pela ciência, que se desdobra na dedicação à formação de jovens pesquisadores e nos serviços à comunidade. Por tudo isso, a Psicologia brasileira será eternamente grata pelos seus ensinamentos e entusiasmo. (comunicação pessoal)

\section{REFERÊNCIAS}

Almeida, S. S., \& de Oliveira, L. M. (1981). Sousalimentation proteique et effects de trois intensités de choc sur 1 aquisition et l'extinction de responses d'evitement. Médecine et Nutrition, 17, 459-466.

De Oliveira, L. M., \& Almeida, S. S. (1985). Effects of malnutrition and environment on the acquisition and extinction of avoidance behavior in rats. Physiology and Behavior, 34, 107-113.

De Oliveira, L. M., \& Graeff, F. G. (1972). Comparison between the effects of amphetamine and apomorphine on operant behavior. European Journal of Pharmacology, 18, 159-165.

De Oliveira, L. M., \& Levitsky, D. A. (1979). Efeitos dos choques a intervalos variáveis sobre o comporta- mento alimentar de ratos subnutridos. Cadernos de Pesquisa, 29, 111-124.

Graeff, F. G., \& de Oliveira, L. M. (1975). Influence of response topography on the effects of apomorphine on operant behavior of pigeons. Psychopharmacologia, Berlim, 41, 127-132.

Keller, F. S. (1996). Report on the Brasilia Plan. Psicologia: Teoria e Pesquisa, 12, 193-197. 